\title{
Correction to "Pharmacological Characterization of Apraglutide, a Novel Long-Acting Peptidic Glucagon-Like Peptide-2 Agonist, for the Treatment of Short Bowel Syndrome"
}

In the above article [Hargrove DM, Alagarsamy S, Croston G, Laporte R, Qi S, Srinivasan K, Sueiras-Diaz J, Wiśniewski K, Hartwig J, Lu M, et al. (2020) J Pharmacol Exp Ther, 373: 193-203; DOI: https://doi.org/10.1124/jpet.119.262238], a typographical error was found in the sequence describing glepaglutide. The error appears in Table 1 in position 1 . The correct aminoacid should be an $\mathrm{H}$ (histidine) and not an $\mathrm{F}$ (phenylalanine). The correct sequence is:

\section{H G E G T F S S E L A T I L D A L A A R D F I A W L I A T K I T D K K K K K K NH2}

This sequence corresponds to the published sequence of glepaglutide. The amino-acid sequence is the only element required to identify a peptide; salt forms are not relevant in this context.

The authors verified the accuracy of the sequence used in their experiment by reexamining a) the original Ferring Study Report and b) the analytical results that accompany the batch release for the compound that was used in the study. The correctness of the sequence in this study was confirmed by the synthesizer log sheet and confirmed by MS analysis. These data were provided to the journal.

The authors thank Jolanta Skarbaliene, Bjarne Due Larsen, Mark Berner-Hansen, and Adam Steensberg of Zealand Pharma A/S for discovering this error and bringing it to the attention of the authors and the journal. The authors apologize for any inconvenience it may have caused. With the correction of the typographical error, the authors stand behind the data as published and the integrity of the findings. They aver that the conclusions of the article remain intact.

The HTML and PDF versions of the article have been corrected. 